\title{
Señderos
}

\section{Reflexiones sobre los métodos de la investigación educativa}

\author{
Reflections on the methods of educational research
}

\begin{abstract}
Autora:
Laura María Giraldo Urrego ${ }^{1}$

Recibido: 21 de febrero 2018

Aprobado: 1 de abril 2018
\end{abstract}

\section{Resumen}

En el presente trabajo se reflexiona críticamente en torno los métodos de la investigación educativa desde el enfoque cualitativo, se discuten algunas complicaciones que acarrea la construcción del conocimiento desde estaperspectivayque se relacionan con su legitimación en la comunidad científica. Las producciones cuantitativas no padecen de problemáticas relacionadas con la credibilidad y objetividad en sus procedimientos, y, como corolario, la ciencia que se crea a partir de parámetros cuantitativos resulta ser por definición legítima. Como conclusión, se plantea que ningún método de investigación debe pretender "capturar" verdades científicas absolutas, pues no es este el propósito de la investigación educativa.

Palabras clave: investigación, enfoque científico, método científico, ciencia. 


\begin{abstract}
In the present work, we critically reflect on the implications of thinking the research from the qualitative approach by discussing some of the complications involved in the construction of knowledge from this approach, and which are related to its acceptance in the scientific community. Quantitative productions do not suffer from problems related to credibility and
\end{abstract}

objectivity in their procedures, and as a corollary, science that is created from quantitative parameters, proves to be by law legitimate. In conclusion, it is suggested that research methods should not aim to "capture" absolute scientific truths, since this is not the purpose of educational research.

Keywords: resarch, scientific approach, scientific method, science.

\section{Introducción}

La investigación, ya sea cualitativa o cuantitativa, es una actividad científica que provee los fundamentos para los informes y las representaciones del «Otro». Denzin y Lyncoln, 2005

La investigación en el ámbito de la educación no es el resultado de un proceso lineal y progresivo, elementos de carácter histórico, en términos políticos, económicos y sociales, la han conducido por distintas vías sin que haya llegado a ninguna suerte de "estado ideal" o no, que denote por sí misma una idea de progreso (Kuhn, 2004, p. 318). Se hace necesario entonces, antes de avanzar en los enfoques adoptados por la investigación educativa, acotar unas breves referencias sobre la ciencia y la investigación. Conocer, construir y saber son procesos inherentes a la idea de ser humano, que lejos de decantar en un permanente avance que va perfeccionando cada vez más a la humanidad, no en pocas ocasiones, esta necesidad y afán por “devorarse al mundo" o "desvelarlo", ha procurado esclavitud, colonizaciones y guerras (Denzin y Lyncoln, 2005). Sin ánimo de obnubilar el panorama, este proceso de conocer, construir y saber, que hoy llamamos Ciencia, ha posibilitado, a pesar de todo, múltiples maneras de ver el mundo, diversas comprensiones de la existencia que, por lo menos en el ideal, han propendido por la mejora de las relaciones humanas. La Ciencia, entonces, es asumida como una actividad específica que se ha dado a lo largo de la historia, pero que en cada época se ha manifestado con valores cognitivos y características propias asumidas por la comunidad científica (Kuhn, 2004).

Ahora bien, el modo mediante el cual se llega a la Ciencia ha sido desde hace mucho tiempo la investigación. Esta última implica el permanente preguntar sobre el porqué, para qué y el cómo de determinado fenómeno, en otras palabras, investigar comienza con la indagación exhaustiva sobre las cosas (Pulido, Ballén y Zúñiga, 2007). Etimológicamente hablando, investigar viene del latín "in” (en) y "vestigare" (pos de la huella de), lo que implica la búsqueda de pistas y vestigios, que, históricamente, 
no se ha efectuado necesariamente bajo métodos elogiosos o loables, toda vez que múltiples han sido las formas de socavar el conocimiento, formas que, por cierto, han ido en contra de muchas voluntades humanas.

Entre tanto, la investigación ha dado origen a diversas formas de hacer lo que llamamos Ciencia, es decir, "conocer, problematizar algún aspecto de la realidad" (Pulido et al., 2007, p. 11). De un lado, durante el siglo XVIII imperó una concepción de ciencia basada en la obtención de certezas y verdades sobre la realidad, sin mencionar que esta última podía ser “capturada” y más que eso, controlada; se trataba de un enfoque cuantitativo que buscaba una medición sistemática del conocimiento. Contraria a la anterior, y gracias a una serie de acontecimientos históricos, a finales del siglo XIX, en el área de la antropología, surgió la investigación de corte cualitativo, misma que se ocultó bajo las sombras del tecnicismo cuantitativo después de la Segunda Guerra mundial. No es sino a partir de los años sesenta que la investigación cualitativa resurge y toma protagonismo en la tarea de construir conocimiento. De ahí que comenzara una larga contienda entre lo cuantitativo y lo cualitativo, pues mientras los primeros desacreditaban la objetividad y cientificidad que tenían los estudios sobre grupos humanos, los segundos realzaban la virtud interpretativa que posibilitaban los fenómenos (Denzin y Lincoln, 2005; 2012).

Antes de seguir, se hace necesario conceptualizar aquello que significa e implica investigar de forma cualitativa. En primer lugar, debe precisarse que la investigación cualitativa no ha sido llevada a cabo de la misma manera a lo largo del tiempo, esto es, que sus métodos han sido variados, toda vez que se ha concebido de una manera u otra, dependiendo de la época. Actualmente, hay consenso en cuanto a entenderla como una forma de identificar la naturaleza subyacente a las realidades, sus bases dinámicas, las que dan razón plena de su comportamiento y manifestaciones (Martínez, 2006). Vale la pena destacar la conceptualización que hacen Denzin y Lincoln (2012) sobre la investigación cualitativa, en tanto la identifican como

una actividad situada que ubica al observador en el mundo. Consiste en una serie de prácticas materiales e interpretativas que hacen visible el mundo y lo transforman, lo convierten en una serie de representaciones que incluyen las notas de campo, las entrevistas, las conversaciones, las fotografías, las grabaciones y las notas para el investigador. [...] implica un enfoque interpretativo y naturalista del mundo. (Denzin y Lincoln, 2012, p. 48)

Asimismo, Denzin y Lincoln (2012) refieren ocho periodos por los que la investigación cualitativa ha atravesado, a saber: el tradicional de 1900-1950, el modernista (1950-1970), el desdibujamiento de los géneros (1970-1986), la crisis de la representación (1986-1990), el posmodernismo (1990-1995), la investigación posexperimental (1995-2000), el presente de las luchas metodológicas (2000-2004) y el futuro fracturado (2004 a la fecha). Estos periodos, enmarcados entre el siglo XX y el presente, logran cobijar los distintos enfoques metodológicos que se han llevado 
a cabo desde la investigación cualitativa, de hecho, es posible que el surgimiento de uno no suponga necesariamente la desaparición de otro.

Grosso modo, la investigación, desde el enfoque cualitativo o cuantitativo, "implica procesos científicos, que actúan como base para fundamentar los informes y las comprensiones sobre el Otro" (Denzin y Lincoln, 2012). Y a pesar de que la investigación cualitativa ha atravesado diversos momentos históricos y por ende variabilidad de métodos, puede decirse que cada uno de ellos continúa hoy vigente y coexisten en la comunidad científica. Actualmente, la investigación cualitativa está compuesta por un sinnúmero de producciones académicas que siguen en aumento, gracias a la convergencia de profesionales investigadores de múltiples disciplinas del conocimiento (Álvarez, 2003).

Ejemplo de ello son la multitud de revistas científicas de corte cualitativo y de alto impacto, entre ellas, Action Research International; Educational Insights; Electronic Journal of Sociology, y Qualitative Report, entre muchas otras. Asimismo, existe diversidad de congresos, seminarios y conferencias que se ofrecen alrededor del mundo sobre investigaciones cualitativas recientes que refieren temas polémicos en diferentes contextos. Así, "la investigación cualitativa en la actualidad constituye un paradigma legítimo que está creciendo y encontrando cada día mayores aplicaciones y utilidades" (Álvarez, 2003, p. 37).

A pesar de todo este bagaje teórico, cuestiones como la validez, fiabilidad y confiabilidad de los resultados obtenidos siguen siendo un problema vigente de la investigación cualitativa. Cristina Moral (2006) expone uno de los panoramas recientes de la investigación cualitativa, y menciona que en la publicación "Estudios Cualitativos en Educación a Principios del Siglo 21", se hace una seria denuncia frente a las reformas que hace poco se han estado introduciendo en la investigación educativa propiamente, al redefinirse desde indicadores netamente positivistas.

Puede decirse que lo anterior vendría a significar una suerte de retorno al pasado con aires de posmodernismo. Simultáneamente, esta situación pone nuevamente en tensión los paradigmas cualitativo y cuantitativo, evidenciando, al parecer, una suerte de 'guerra fría donde un método busca imponerse sobre el otro, o por lo menos mostrarse como el más valido. Por todo ello actualmente resurgen intentos por ratificar los criterios de veracidad que han amparado la investigación cualitativa desde sus inicios, en palabras de Sandín:

Aunque la investigación cualitativa posee una dilatada historia en el seno de las disciplinas sociales, en las dos últimas décadas ha tenido que luchar, no tanto, por abrir espacios de indagación y reflexión que admitieran otras formas de acercamiento a la realidad, cuanto por el reconocimiento de su legitimidad. (2000, p. 224). 
Y es que el problema sigue siendo, no tanto su uso en el 'mercado académico', sino su importancia dentro de él a la hora de impactar socialmente. La hegemonía de las 'ciencias duras', medibles y cuantificables, frente a las 'ciencias blandas' subjetivas y relativas, se ve reflejada en la esfera social. Como ilustración, en el sector laboral, la mayoría de las carreras profesionales que impliquen valoraciones en términos numéricos, es doble o triplemente mejor remunerada que una carrera profesional inscrita dentro de las ciencias humanas (Denzin y Lyncoln, 2012), aun suponiendo la misma duración temporal de formación. Como corolario, el derecho, la medicina y las ingenierías son los empleos mejor retribuidos económicamente, por lo menos en Colombia (Borjas y Vílchez, 2009; Denzin y Lyncoln, 2012; Roedig, 2012; Upegui, 2015). La cuestión de los estipendios no es un asunto que deba tomarse a la ligera ni analizarse únicamente desde el punto de vista mercantil, sino que necesariamente implica una mirada crítica desde la valoración social y simbólica que se otorga a estas ciencias.

Ahora bien, paradójicamente, el aspecto más loable y plausible de la investigación cualitativa, a saber, la interpretación, es precisamente su punto más débil ante la mirada del control cuantitativo. La controversia entre estos dos enfoques consiste en que cada uno apela a deslegitimar el punto fuerte del otro. En otras palabras, mientras la teoría cualitativa sufre por su grado de subjetividad que le impide generalizar y aplicar sus constructos a múltiples contextos, la teoría cuantitativa padece el hecho de llevar a términos numéricos las situaciones humanas, mismas que pretende objetivar sin percatarse del inevitable desbordamiento de la realidad (Schwab, 1996). Cabrero y Richart (1996) también hacen referencia al anterior dilema cuando describen algunas de las mitificaciones más extendidas sobre este terreno: "la investigación cualitativa es no científica, subjetiva, de generalidad limitada y blanda, y la investigación cuantitativa es superficial, extrañada de la realidad, sometida a un empirismo estéril, y duras" (p. 213).

La polémica que se remonta al siglo XIX, versa entonces sobre los modos de concebir la realidad; esta confrontación entre los exponentes de las ciencias 'duras' y los representantes de las ciencias 'blandas', por el modo más lícito de presentar la existencia, sigue hoy vigente, en ocasiones flexible una con la otra, pero otras veces radicalizadas en sus posturas (López, 2005). A pesar de este eterno enfrentamiento entre paradigmas, hay quienes apelan a un acoplamiento entre ambas.

No es de desconocer que los dos paradigmas comparten la fragilidad de lo subjetivo, toda vez que ambos parten del propio ser humano, lo cual, por definición, deja por fuera la objetividad. Es plausible que no hay nada más subjetivo que unas cuantas personas aisladas, experimentando, manipulando y adecuando en un laboratorio sus propios intereses y motivaciones, y es que "lo subjetivo es lo influido por el juicio humano y, desde esa acepción, tanto lo cualitativo como lo cuantitativo son subjetivos" (Cabrero y Richart, 1996, p. 214). Asimismo, nótese que lo que tiene que ver con la identificación de las emociones, los valores, los sentimientos y las concepciones es cuantitativamente medido con técnicas psicométricas y sociométricas, por lo cual no es exclusivo de la esfera cualitativa el abordaje de 
la subjetividad (Cabrero y Richart, 1996). Otro eje del debate mantiene que los métodos cualitativos son básicamente exploratorios, inductivos, frente a los métodos cuantitativos, que son de utilidad confirmatoria y de naturaleza hipotéticodeductiva. Sin embargo, es menester destacar los argumentos de los dos 'padres' de la investigación cualitativa y creadores de la teoría fundamentada, a saber, Glaser y Strauss (1967):

There is no fundamental clash between the purposes and capacities of qualitative and quantitative methods or data. What clash there is concerns the primacy of emphasis on verification or generation of theory to which heated discussions on qualitative versus quantitative data have been linked historically. We believe that each form of data is useful for both verification and generation of theory, whatever the primacy of emphasis. Primacy depends only on the circumstances of research, on the interests and training of the researcher, and on the kinds of material he needs for his theory (pp. 17-18).

Lo señalado por Glaser y Strauss resulta coherente en tanto es posible que el dilema entre lo cualitativo y lo cuantitativo sea más una cuestión de relaciones de poder que realmente un problema sustancial, incluso epistémico, de validez. Puede decirse que para una situación o problemática concreta existen varias formas de abordarse, pero es en consideración de los fines que uno u otro método le vendría mejor, siendo así la manera más fiable y válida de solucionar o comprender el problema. De hecho, en el mejor de los casos las distintas metodologías de intervención podrían complementarse, no necesariamente alienarse, pero sí contribuirse mutuamente.

Cuando se pretende ser partidario exclusivo de un método u otro, vale tomar en cuenta el hecho de que muchos de los investigadores de determinado campo se basan no solo en el resultado de sus análisis para generar teoría, sino que también aprovechan los 'descubrimientos' de su 'contrincante' para apoyar y respaldar sus propias cuestiones. Lo anterior no quiere decir que uno u otro método tengan que cuidarse de no inmiscuirse en el camino del otro. Por el contrario, se presenta como un indicio de que ambos pueden soportarse el uno al otro (Glaser y Strauss, 1967, p. 18).

Pasando a otro punto, la investigación cualitativa, como ya se ha dicho, nunca ha sido un enfoque lineal y concreto, toda vez que en el transcurrir del tiempo han surgido nuevas maneras de ahondar en las realidades sociales, y el enfoque cualitativo ha desplegado un abanico de posibilidades para beneficio de los investigadores y sus intereses particulares. La variedad de metodologías cualitativas obedece a su complejidad y necesidad de configurar un orden conceptual y epistémico que aclare a la comunidad científica los fines y métodos de la investigación (Sandín, 2003). Por lo anterior, realizar una investigación cualitativa, sin un previo análisis de la metodología que se va a implementar con base en los objetivos del estudio, queda fuera de contexto y sin respaldo epistemológico que otorgue coherencia y seriedad tanto a la investigación como al investigador. Por esta misma razón, Canales y Peinado (1995, citados en Sandín, 2003) afirman que "sin epistemología y metodología que la sustente, una 
técnica de investigación es apenas un confuso conjunto de procedimientos canónicos. Esta afirmación válida para cualquier técnica, adquiere especial relevancia en el caso de las llamadas técnicas cualitativas” (p. 287).

Por otro lado, y no necesariamente en contra de las exposiciones de Sandín (2003), pero sí con ánimo de relativizar en cierta medida la postura de Canales y Peinado, autores como Hernández, Fernández y Baptista (2006) hacen hincapié en que los lindes entre un método y otro de investigación cualitativa suelen ser difusos y de hecho alienables:

Las fronteras entre los diseños cualitativos realmente no existen. Por ejemplo, un estudio orientado por la teoría fundamentada abarca elementos narrativos y fenomenológicos. Una investigación-acción puede generar codificación axial (teoría fundamentada) cuando analiza entrevistas realizadas a participantes respecto a cierto problema de interés. Creemos que el estudiante no debe preocuparse tanto sobre si su estudio es narrativo o etnográfico, su atención más bien tiene que centrarse en realizar la investigación de manera sistemática y profunda, así como a responder al planteamiento del problema.

(Hernández et al., 2006, p. 713)

De todas formas, existe un consenso en el que destacan como principales algunas metodologías o tradiciones (en términos de Sandín) de la investigación cualitativa a saber: la Investigación- acción, la Etnografía, la investigación Biográfico-Narrativa, la Teoría Fundamentada, el Estudio de Casos y la Investigación Evaluativa. Todas ellas constituyen formas de adentrarse en la comprensión de las realidades sociales y educativas, comprenden diversos fines, instrumentos y modos de accionar. El propósito en este punto no es ahondar en cada uno de los diseños cualitativos, sino acotarlos de forma lacónica pero rigurosa, destacando además aspectos que resulten de interés.

Pues bien, comenzando por la Investigación-acción, puede decirse que es un diseño que busca indicios que propicien la transformación de una realidad concreta, procurando su mejora desde un enfoque crítico y contextual. Su propósito no se basa en la generación de teoría sino, más bien, en la solución de problemas, en formar de manera continua a los participantes, en introducir nuevos modelos de enseñanza, en crear un puente de comunicación entre investigadores y participantes y en afrontar situaciones problémicas del aula (Cohen y Manion, 1985, citado en Sandín, 2003). Una definición que brindan Kemmis y MacTaggart (1988) apunta a la investigación-acción como:

A form of collective, self-reflective inquiry that participants in social situations undertake to improve: (1) the rationality of justice of their own social or educational practices; (2) the participants' understanding of these practices; and the situations in which they carry out these practices. Group of participants can be teachers, students, parents, workplace colleagues, social activists or any other community members - that is, any groups with a shared concern and 
the motivation and will to address their shared concern (citados en Altrichter, Kemmis, MacTaggart y Skerritt, 2002, p. 125).

Según se deja entrever, la transformación que confiere la investigación-acción no es una que brinda el investigador a los participantes con base en diagnósticos o datos emergentes. Por el contrario, es aquella que los miembros de la comunidad educativa son capaces de emprender por sí mismos a través de la reflexión y orientación del investigador, partiendo sobre todo de un ejercicio de libertad y voluntad.

Por su parte, la Investigación Etnográfica alude a un conocimiento profundo y exhaustivo de las formas de existencia de un grupo social determinado. Por estas mismas razones, la etnografía tiene lugar dentro de la educación, en tanto modo de acercamiento comprensivo a la realidad escolar. Pero antes de examinar la etnografía dentro del escenario educativo, se presenta continuación una visión más general. Según Clifford Geertz, este diseño de investigación

[...] consiste en lanzarnos a una desalentadora aventura cuyo éxito sólo se vislumbra a lo lejos; tratar de formular las bases en que uno imagina, siempre con excesos, haber encontrado apoyo, es aquello en que consiste el escrito antropológico como empeño científico. No tratamos (o por lo menos yo no trato) de convertirnos en nativos (en todo caso una palabra comprometida) o de imitar a los nativos. Sólo los románticos o los espías encontrarían sentido en hacerlo. Lo que procuramos es (en el sentido amplio del término en el cual este designa mucho más que la charla) conversar con ellos, una cuestión bastante más difícil, (y no sólo con extranjeros) de lo que generalmente se reconoce. (Geertz, 1990, p. 27)

Claramente la conceptualización de Geertz alude a una manera global de entender este diseño y, sobretodo, de desmitificar algunos presupuestos de los que se suele partir a la hora de investigar bajo el diseño etnográfico, como es el caso de volverse uno más dentro de una comunidad. Pero, ¿qué hay de la etnografía educativa? Pues, dado que este diseño no está predeterminado con una serie de pasos jerárquicos para llevarse a cabo, son diversas las maneras de hacer etnografía en educación (Sandín, 2003). Lo que sí debe tenerse claro son los objetivos de la investigación, mismos que sustentan el modo de proceder en el estudio. No obstante, se debe tener cautela con esta suerte de libertad metodológica, dado que se podría confundir u obnubilar el sentido de los instrumentos utilizados. Por ello Wilcox (1993) aclara que

[...] es bastante más que una serie de técnicas de recogida de datos que se puedan describir y adoptar con facilidad. Su soporte conceptual y su modo de utilización reflejan básicamente las características de la disciplina dentro de la que fue concebida y desarrollada. Etnografía no es sinónimo de observación participante, trabajo de campo o investigación cualitativa. Una comprensión más profunda de la etnografía requiere así mismo una comprensión de la disciplina de la Antropología. (Wilcox, 1993, citado en Axpe, 2003, p. 16). 
Por tanto, este diseño no es algo que deba tomarse a la ligera por el hecho de no comprender una serie de fases sistemáticas de aplicación. Precisamente, es lo anterior lo que torna la etnografía en educación como algo complejo que requiere desentrañar los presupuestos de los que se parte, de modo que el mismo investigador marque las pautas para realizar la investigación, proceso que supone una mayor rigurosidad en la elaboración del esquema.

Por otro lado, frente a la investigación Biográfico-Narrativa, es importante acotar que este diseño de investigación entró en apogeo en los años setenta, en aras de recuperar la memoria individual y colectiva de distintos grupos sociales migratorios en Estados Unidos. Así, pues, la narrativa se basa básicamente en los relatos, historias e informes de experiencias personales para aportar al ámbito educativo desde la voz y las vivencias del sujeto particular. En la investigación narrativa también se estudian fenómenos y problemáticas tanto sociales como educativas, entendidas como "textos, cuyo valor y significado, primariamente, vienen dados por la autointerpretación que los sujetos relatan en primera persona, donde la dimensión temporal y biográfica ocupa una posición central" (Bolívar, 2002, p. 6).

Dentro de este diseño destacan principalmente los documentos personales (Pujadas, 1992, citado en Sandín 2003) como diarios y objetos íntimos, las cartas, las fotografías y los filmes, entre otros. Y, de otro lado, Las historias de vida cobran especial importancia en este diseño, toda vez que ellas no solamente relatan un hecho particular de una persona, sino que incluso dan cuenta de todo un paradigma que le subyace, de unas tradiciones, una época y unos valores que se enmarcan en posturas políticas (Denzin, 2012). De igual modo, para Ruíz e Ispizua (1989, citados en Sandín, 2003), es el método por excelencia mediante el cual el investigador aprende el modo en que el otro construye y proyecta su existencia.

Ahora bien, la investigación narrativa en educación, tiene especial visibilidad a través de la obra de Philip Jackson, La vida en las aulas, donde recrea anécdotas y vivencias con el profesorado y los alumnos. En esta obra se relata cómo no solamente sus estudios impactan en las experiencias de los maestros y estudiantes, sino que también deja entrever la forma en que este diseño de investigación transforma la propia experiencia del investigador, lo cual lo sitúa en una constante disertación consigo mismo:

Me enseñó que lo familiar y lo ordinario eran barreras que había que penetrar y que un modo bastante mecánico de lograrlo consistía en desligarse uno mismo de su semiparticipación en la actividad en curso y dedicarse a observar lo que sucedía como si estuviera muy distante o como si viera una película sin sonido ni subtítulos. [...] me mostraba verdaderamente ambivalente acerca de mi supuesta neutralidad, aunque no siempre lo reconociera entonces ni siquiera en mi fuero interno. (Jackson, 1992, pp. 32-38) 
Otro diseño relevante, aunque no tan usado en educación, es la Teoría Fundamentada, una metodología cualitativa conocida por posibilitar la generación o creación de teoría a partir de la investigación, que recoge además aspectos fundamentales del interaccionismo simbólico, procurando la generalización de ideas a partir de los resultados obtenidos y no desde hipótesis previamente establecidas (Sandín, 2003). Su principal objetivo es construir nuevos marcos conceptuales, pero que se puedan generalizar. En palabras de los creadores de esta metodología:

Most writing on sociological method has been concerned with how accurate facts can be obtained and how theory can thereby be more rigorously tested. In this book we address ourselves to the equally important enterprise of how the discovery of theory from data-systematically obtained and analyzed in social research-can be furthered. We believe that the discovery of theory from data-which we call grounded theory-is a major task confronting sociology today, for, as we shall try to show, such a theory fits empirical situations, and is understandable to sociologists and layman alike. Most important, it worksprovides us with relevant predictions, explanations, interpretations and applications. (Glaser y Strauss, 1967)

La posición de los autores constata una fidedigna credibilidad en su trabajo como una base que soporta la premisa según la cual la construcción de teoría está al alcance de cualquier persona. Esto entra en conflicto con las posturas positivistas del conocimiento, bajo las cuales solo se hace teoría a través del método científico. Lo anterior configura la Teoría Fundamentada positivamente, en tanto que no se muestra como excluyente, y no deja de lado la rigurosidad en la investigación. Sin embargo, y no solo con este método, siempre habrá que caminar firme, pero con cautela cuando se trata de 'crear' o 'descubrir' una teoría, comenzando por el hecho de que éstas pretenden en principio (bien sea desde un enfoque cualitativo o cualitativo) generalizar en cierta medida los resultados que construyen.

A propósito de esta cuestión, Tadeu da Silva hace una crítica a esta concepción que, sin ánimo de debatir la filosofía que soporta la teoría fundamentada, valdría la pena traerla a colación, en la medida en que es quizás un asunto que corre el riesgo de olvidarse en el momento en que la figura 'Teórica' deslumbra la mirada del lector o investigador:

En general, en la noción de teoría está implícita la suposición de que la teoría "descubre" lo "real", de que hay una correspondencia entre la "teoría" y la "realidad". De una forma u otra, la noción involucrada es siempre representacional, especular, mimética: la teoría representa, refleja, espeja la realidad. Es una representación, una imagen, un reflejo, un signo de la realidad que -cronológica, ontológicamente - la precede. (Tadeu, 1999, p. 3)

Siempre es oportuno reflexionar sobre este tipo de cuestiones, que, aunque parecieran alejadas del método concreto de una investigación, tienen más relación con él del que aparentan, toda vez que el conjunto de presupuestos que configuramos respecto a un 
fenómeno, tema o cuestión suele ser el foco que guía nuestro accionar. En esa medida, nuestros esquemas mentales, producto de las creencias y experiencias que, a su vez, se derivan de aquello que legitimamos como verdad, repercuten en la vida social de manera trascendental. Por ende, es necesario tener claridad sobre el nivel de validez que otorgamos a determinada 'teoría', de modo que esta última no actúe como veto a fenómenos que se no se enmarquen dentro de sus generalizaciones.

Con relación al Estudio de Casos, es necesario acotar que muchas veces es catalogado como método de investigación en sí mismo y otras tantas como parte de las estrategias metodológicas de un estudio (Denzin y Lincoln, 2006). De todas formas, en la práctica investigativa, tanto una como otra forma de asumirlo se presentan como legítimas ante la comunidad científica. Grosso modo, el Estudio de Caso se basa en la investigación de una realidad o fenómeno concreto, caracterizado por ser particularista, sistémico, basado en la descripción e inductivo (Pérez, 1994, citado en Sandín, 2003). Frente a este particularismo, es necesario reflexionar cuántas veces el ser humano analiza y focaliza sus sentidos hacia un objeto o persona que aparentemente es común e insignificante, puesto que normalmente se vuelca la atención sobre algo extraordinario u ostentoso. No obstante, el estudio de casos se basa precisamente en la particularidad, en el análisis minucioso y detallado de lo singular para poder comprender su complejidad (Stake, 2007).

Por lo anterior, es factible que la filosofía tendría mucho que ver como parte del proceso de la investigación en un Estudio de Casos. Quizás la pregunta que surja sea ¿qué tiene que ver una cosa con la otra? Pues bien, se trata de que hay una imposibilidad por construir de la nada, ya que se requiere partir de algo para generar nuevas cosas, el problema radica en hallar ese algo, en encontrar un asunto tan meritorio y nunca antes visto, que sea merecedor de la atención humana. Posiblemente esta sea una labor agobiante... o no, depende. Al respecto, vale la pena propender por una actitud o asombro filosófico que coadyuve a encontrar lo deseado, incluso si aún no se conoce. El asombro filosófico que se refiere es de aquel que se sorprende con la mismidad permaneciente al fenómeno u objeto, el mismo que es recurrente en el tiempo, en el espacio y en el ser, pero que se convierte siempre en único, nuevo e irrepetible cada vez que el investigador lo mira con un lente nuevo.

La actitud no filosófica y la filosófica se dejan separar con gran claridad (no existiendo apenas formas transitorias). La primera acepta todo lo que ocurre en su contexto tanto general como natural y se asombra, en todas las ocasiones, solo por el contenido especial según el cual el suceso hoy aquí se diferencia del suceso ayer allí. Mientras que, por el contrario, la segunda concibe justamente los rasgos comunes de todas las vivencias, que caracterizan de forma general lo hallado. Si, se podría decir que el hecho de vivir y encontrar se concibe como primer y más profundo motivo de perplejidad. Me parece que este segundo tipo de asombro, cuya existencia se encuentra fuera de toda duda, es el mismo algo muy asombroso. (Schrodinger, 1998, p. 27) 
Este asombro o actitud filosófica se relaciona con el Estudio de Casos, dado que su metodología implica observar fenómenos que para algunos podrían resultar triviales o que son "poco significativas" en el ámbito científico, ya sea porque son muy específicos y no se pueden generalizar (Flyvbjerg, 2006), o porque no se les considera de alto impacto social. Como agravante, no son valorados lo suficiente si el caso no es lo bastante llamativo o inusual dentro del campo investigativo. Pero lo que se pretende realzar aquí es que cualquier caso o fenómeno es susceptible de análisis y puede ser atrayente e importante para la sociedad, puesto que estas dos últimas cualidades son atributos otorgados por el ser humano; no son propias de ningún objeto o fenómeno en sí mismo, sino que son los sujetos quienes tienen la facultad de realzar o denigrar el valor de cualquier objeto, persona o situación, y para ello se requiere de esta actitud y mirada filosófica. En relación con este punto neurálgico de los estudios de casos, Flyvbjerg, estudioso de las ciencias sociales, afirma:

One can often generalize on the basis of a single case, and the case study may be central to scientific development via generalization as supplement or alternative to other methods. But formal generalization is overvalued as a source of scientific development, whereas "the force of example" is underestimated. (2006, p. 228)

Ahora bien, un último diseño del que se hará mención es la Investigación Evaluativa, misma que busca, concretamente, evaluar programas educativos a fin de determinar si estos son eficaces y pertinentes, que a su vez posibiliten la toma de decisiones pertinentes en la práctica educativa (Latorre et al., 1996, citado en Sandín, 2003). Por otro lado, Escudero (2003) afirma que, en investigación evaluativa, de hecho, más que hablar de metodologías para llevarla a cabo, se puede hablar de enfoques, ya que son distintas las maneras de llevarla a consecución, algunas desde miradas más instrumentales y otras desde ópticas tradicionales. En materia de educación, vale destacar un momento histórico crucial para la investigación evaluativa, Escudero señala que en los años sesenta se pueden diferenciar dos modelos de procedimiento:

We can qualify one as evaluation orientated toward individuals, fundamentally students and teachers. The other level is that of evaluation orientated to decision making on the "instrument" or "treatment" or educational "program." This last level, also impelled by the evaluation of programs in the social environment, will be the basis for the consolidation in the educational field of program evaluation pro and of evaluation research. (Escudero, 2003)

Para el caso del proyecto social educativo, mencionaré las características principales que Escudero (2003) recopila, puesto que las considera necesarias a la hora de diseñar un programa evaluativo. Lo primero a tener en cuenta es el objeto o propósito de la investigación evaluativa y, en consonancia con ello, quiénes serían los implicados o beneficiados. En segundo lugar, habría que definir qué aspectos se busca evaluar con su respectiva justificación, y una vez establecidos delimitar qué información se requiere y cómo se recogerá. Asimismo, se elegirá el o los métodos de análisis, así como las personas que harán parte de ese proceso, el cual debe ser secuenciado valiéndose de 
informes y sistematizaciones. Finalmente, habrán de exponerse tanto las limitaciones del programa evaluado como las del propio modelo de evaluación (metaevaluación).

Es menester resaltar que la Investigación Evaluativa puede hacerse desde una mirada cuantitativa o cualitativa, en función de los intereses particulares de la comunidad que requiere la evaluación. Lastimosamente, los métodos cualitativos son los menos utilizados en este 'mercado' académico, dado que requieren mucho más tiempo y posiblemente recursos para llevarse a cabo. Por ende, los test y pruebas paramétricas imperan dado el grado de demanda a la hora de evaluar programas educativos.

Ahora bien, una vez comentadas las metodologías de investigación cualitativas, se retomará nuevamente lo que supone investigar bajo el lente cualitativo, pero ahora desde el punto de vista de uno de los instrumentos más relevantes para la recogida de datos: Los grupos de discusión. Este instrumento se configura como forma dialógica de recoger y analizar información, partiendo del sujeto y su perspectiva sobre un fenómeno o problemática determinados. El procesamiento de datos provenientes de los grupos de discusión o Focus Group (en términos anglosajones), implica necesariamente un enfoque hermenéutico dentro de la investigación, toda vez que este instrumento se basa en una teoría de la interpretación de los símbolos lingüísticos del sujeto a partir de sus relatos narrativos. Remontándonos brevemente a su historia, los grupos de discusión surgen en la década de 1930 como técnica terapéutica para tratar un problema personal, mientras se comparte la experiencia con otros (Gil, 1993). En los años que comprenden la Segunda Guerra mundial los grupos de discusión tuvieron lugar, pero desde el ámbito del marketing, y desde los años ochenta hasta la actualidad pasan a otra faceta, permeándose de la totalidad de las áreas del conocimiento (Llopis, 2004). Su inclusión y resurgimiento en las ciencias humanas tiene que ver con el deseo de éstas de encontrar nuevas vías para recoger información. En palabras de Llopis:

Es la necesidad de resolver cuestiones prácticas, suscitadas en el transcurso de la aplicación de herramientas convencionales de investigación social y de mercados, lo que empuja el nacimiento y desarrollo del grupo de discusión como una práctica de investigación. Una práctica cuya formación no se establece de una vez para siempre, ya que se desarrolla en un proceso de incesante adaptación a las exigencias de las situaciones del estudio. (2004, p. 26)

Ahora bien, propiamente en España y Latinoamérica, los grupos de discusión cobran relevancia bajo los estudios de Ibáñez en psicoanálisis durante los años noventa (Menda y Méndez, 2009). Para este autor, los grupos de discusión se basan en el planteamiento de un tema general y en la escucha activa de la conversación entre el grupo, mientras que, en una tradición más anglosajona, el moderador plantea preguntas que avocan a los participantes a dialogar en torno a ellas (Gil, 1993). A parir de la obra de Rosaline Barbour (2013), básicamente los grupos de discusión se enfocan en la exploración y ahondamiento de un conjunto específico de cuestiones. 
Así, pues, a través de este instrumento el investigador tiene la función de moderar o dirigir el grupo, procurando que la conversación o el debate se encaminen hacia los objetivos del estudio. De cualquier modo, el grupo de discusión aparece como un espacio de reflexión donde se intercambian puntos de vista, opiniones y argumentos.

Como resultado del sustento teórico que ampara los grupos de discusión, hay una legitimación de estos como instrumento idóneo siempre y cuando esté en consonancia con los objetivos y el tipo de participantes de la investigación. Experiencias del profesorado testimonian la recurrencia de situaciones donde se hacen preguntas a un grupo de niños (entre 3 y 6 años) y uno de ellos contesta en primer lugar, los demás suelen adherirse a la primera respuesta, o al menos varios de ellos lo hacen. Lógicamente, esto no sucede siempre, ni en todos los contextos ni con todos los niños, pero siendo un fenómeno que se presenta no pocas veces, tanto en niños como en adultos, debe considerarse a la hora de pretender recoger resultados 'limpios' de un grupo de discusión.

Es factible que el ejemplo anterior no suceda tan a menudo con adultos, pero puede ser que, si bien a los adultos no los convoque la idea de repetir respuestas por falta de imaginación o intimidación, podrían ser otras cuestiones las que les guíen hacia ese tipo de reacciones. Verbigracia, en un grupo de discusión puede presentarse que un participante formule su respuesta igual a la de otros participantes, a razón de una inclinación de la mayoría. Es posible que este fenómeno tenga sentido, quizás por el miedo a contestar algo que vaya en contraposición de lo que aparentemente es la respuesta más obvia, 'normal' o mejor aceptada. Esto debe contemplarse a la hora de establecer grupos de discusión. Recuérdese el famoso experimento de Solomon Asch en la década de 1950, que demuestra cómo, por diversos motivos, un grupo de sujetos puede distorsionar su juicio y, por ende, modificar sus respuestas frente a una pregunta guiándose por lo que piensa la mayoría (Henle, 1961).

La hipótesis de Asch se basaba en establecer que las percepciones de las personas cambiaban bajo una fuerte presión social. No obstante, su idea solamente dio cuenta de que las personas a quienes se les hizo la pregunta experimental contestaron lo que la mayoría por temor a las consecuencias que acarrearía contradecir a los demás; algunos también lo hicieron por la imagen social proyectada, si no mostraba acuerdo con el buen juicio del resto, y una "muestra poco significativa” evidenció haber distorsionado su percepción (Cruces et al., 1991). A lo anterior Asch lo llamó Conformidad grupal, y en un grupo de discusión también se puede presentar este efecto, a pesar, y precisamente, por la confluencia de opiniones, fácilmente un participante puede cambiar lo que piensa en un primer momento respecto a un tema por lo que acotan los demás, o, en el peor de los casos, mantener sus convicciones pero verbalizar ideas contrarias.

En menester señalar que los grupos de discusión no siempre resultan idóneos para cualquier investigación cualitativa, puesto que cada metodología, sea etnográfica, fenomenológica, estudio de caso, etcétera, sugiere los instrumentos más convenientes 
para la recolección de datos. Es el caso, por ejemplo, de la investigación de tipo narrativa, donde las entrevistas suelen ser más pertinentes que los grupos, y no porque los participantes no desplieguen con elocuencia su discurso, sino porque mantener la hilaridad y coherencia de la información proveniente de varias fuentes que confluyen simultáneamente resulta una tarea compleja, tal como refiere Barbour (2013).

Por otra parte, es necesario no dejar a un lado las posibilidades y virtudes del grupo de discusión, comenzando por decir que muchas veces este instrumento posibilita que los participantes de un estudio entren en una zona de tranquilidad, confianza y reconocimiento que les incita a hablar y poner en palabras sus sentires y criterios, cosa que en ocasiones la entrevista individual no logra obtener. Pero lo más loable de este asunto es que la voz y las disertaciones de los participantes son las que esgrimen la base para construir y configurar los saberes que denotan en sus discursos. Puede entablarse un símil con El Banquete de Platón (2013), reunión donde no pocos filósofos se encontraron para discutir cuestiones sobre el amor, y a pesar de que cada participante del banquete emitía un breve discurso sobre un asunto derivado, fuese la homosexualidad, los sentimientos, el eros, etcétera, todos tenían la posibilidad de debatir o apoyar las ideas con sus propios argumentos. Esta analogía tiene lugar en tanto hace las veces de génesis romántica de los grupos de discusión como entornos de construcción de racionalidad conjunta.

Ahora bien, nuevamente se hace un giro hacia el diseño de investigación de tipo narrativo, el cual sin duda implica la inmersión en la compleja profundidad del ser. Hoy por hoy, los estudios narrativos han cobrado fuerza y visibilidad académica, lo que ha decantado en el llamado Síntoma Biográfico (Santamarina y Marinas, 1995, citados en Sandín, 2003). Este indicador convoca a un resurgir del discurso que tradicionalmente estaba velado en la investigación tradicional, pero que actualmente reaparece con fuerza ensanchando su campo en pro de formar una autoconciencia de la sociedad a través de los relatos e historias del sujeto, como manera de reivindicar las experiencias personales (Bolívar y Domingo, 2006).

Pero no hacen falta historias rimbombantes y escandalosas para que la narrativa encuentre su objeto, todo lo contrario, son las vidas de los sujetos comunes y corrientes las que dan lugar al interés del investigador. A propósito de esto, en Vidas minúsculas (obra de la literatura francesa) se relata cómo a través de la vida privada de otras personas, Pierre Michon teje la propia, ensalzando la aparente trivialidad de la existencia de los más pobres y apartados de la sociedad francesa (Murillo, 2014). Esta obra es un ejemplo del potencial narrativo de las historias biográficas de las personas que representan una suerte de Cuarto Estado (obra de Giuseppe Pellizza).

Es así como las historias de vida y las biografías parecen tener, hoy día, una trascendencia renovada, toda vez que hay una exploración a profundidad de los saberes del colectivo social, en contraposición a las grandes estructuras o dispositivos de poder que suelen adquirir protagonismo por su mera existencia. Y es en este escenario investigativo, donde los docentes tienen 
un dilatado campo de acción. La relevancia inherente a la documentación narrativa desde las experiencias escolares por parte de los docentes, se fundamenta bajo el vasto potencial contenido en sus productos o relatos pedagógicos, dado que es una interpretación inedia del mundo escolar desde el punto de vista de sus protagonistas; de los múltiples trayectos de la formación docente. (OEA y AICD, 2005)

A modo de cierre, se hace necesario resaltar que posiblemente la pretensión de la investigación cualitativa, fuere cual fuere su metodología, se basa en deslegitimar, desnaturalizar y poner en cuestión cánones universales; mostrar, explicar y comprender aquello que para algunos resulta insulso, aquello que se escapa de las prescripciones teóricas genéricas. Esto, a fin de cuentas, es una de las bases de la investigación, la pesquisa exhaustiva por los pequeños detalles que devienen en grandes aportaciones a la sociedad.

\section{Referencias}

Altrichter, H., Kemmis, S., MacTaggart, R. \& Skerritt, O. (2002). The Concept of Action Research. The Learning Organization, 9(3), 125-131. Recuperado de http://www.naclc.org.au/cb_pages/files/The\%20concept\%20of\%20Action\%20 research\%20-\%20Kemmis\%2oand\%20McTaggart.pdf [Consultado el 13 de enero de 2018].

Álvarez, J. (2003). Cómo hacer investigación cualitativa: fundamentos y metodología. México: Paidós.

Axpe, M. (2003). La investigación etnográfica en el campo de la educación. Una aproximación meta-analítica. (Tesis doctoral). Universidad de La Laguna.

Barbour, R. (2013). Los grupos de discusión en Investigación Cualitativa. Madrid: Morata.

Bolívar, A. (2002). “¿De nobis ipsis silemus?”: Epistemología de la investigación biográfico-narrativa en educación. Revista electrónica de investigación educativa, 4(1), 1-26. Recuperado de http://www.scielo.org.mx/pdf/redie/v4n1/v4nia3.pdf [Consultado el $10^{\circ}$ de enero de 2018].

Bolívar, A. y Domingo, J. (2006). La investigación biográfica y narrativa en Iberoamérica: campos de desarrollo y estado actual. Forum Qualitative Sozialforschung, 7(4). Recuperado de http://www.qualitative-research.net/ index.php/fqs/article/view/161/357 [Consultado el 10 de enero de 2018]

Borjas, M. I. y Vílchez, C. F. (2009). Ciencias "duras" vs ciencias "blandas". REDHECS: Revista electrónica de Humanidades, Educación y Comunicación Social, 4(7), 195-209. Recuperado de http://publicaciones.urbe.edu/index.php/ REDHECS/article/viewArticle/624/1590 [Consultado el 1. ${ }^{\circ}$ de enero de 2018].

Cabrero, J. y Richart M., M. (1996). El debate investigación cualitativa frente a investigación cuantitativa. Enfermería Clínica, 6(5), 212-217. Recuperado de 
https://www.researchgate.net/publication/266217625_El_debate_investigacion_cualitativa_frente_a_investigacion_cuantitativa [Consultado el 12 de enero de 2018].

Cruces, S. et al. (1991). El fenómeno del conformismo ante situaciones no estructuradas: Confirmación de la hipótesis gestáltica de Asch. Revista de Psicología Social, 6(1), 61-71.

Denzin, N. (2012). Triangulation 2.o. Journal of Mixed Methods Research, 6(2), 80-88. Recuperado de http://journals.sagepub.com/doi/full/10.1177/1558689812437186 [Consultado el $1 .^{\circ}$ de enero de 2017].

Denzin, N. y Lincoln, I. (2012). El campo de la investigación cualitativa. Barcelona: Gedisa.

Escudero, T. (2003). From tests to current evaluative research. One century, the XXth, of intense development of evaluation in education. Relieve, 9(1). Recuperado de http://www.uv.es/RELIEVE/v9n1/RELIEVEv9n1_1eng.pdf [Consultado el $1 .^{\circ}$ de enero de 2017].

Flyvbjerg, B. (2006). Five misunderstandings about case-study research. Qualitative Inquiry, 12(2), 219-245. Recuperado de http://flyvbjerg.plan.aau. $\mathrm{dk} /$ Publications2006/0604FIVEMISPUBL2006.pdf [Consultado el 30 de enero de 2017].

Geertz, C. (1990). La interpretación de las culturas. Barcelona: Gedisa. Recuperado de https://antroporecursos.files.wordpress.com/2009/03/geertz-c-1973-la-interpretacion-de-las-culturas.pdf [Consultado el 1. ${ }^{\circ}$ de enero de 2018].

Gil, J. (1993). La metodología de investigación mediante grupos de discusión. Enseñanza, (10-11), 199-214. Recuperado de https://idus.us.es/xmlui/bitstream/ handle/11441/16848/file_1.pdf?sequence=18isAllowed=y [Consultado el $1 .^{\circ}$ de enero de 2018].

Glaser, B. \& Strauss, A. (1967). The Discovery of Grounded Theory: Strategies for Qualitative Research. Chicago: Aldine Pub. Co. Recuperado de http://www.sxf. uevora.pt/wp-content/uploads/2013/03/Glaser_1967.pdf [Consultado el 11 de enero de 2018].

Henle, M. (1961). Documents of Gestalt Psychology. Berkeley: University of California Press.

Hernández, R., Fernández, C. y Baptista, P. (2006). Metodología de la investigación (4. ${ }^{\mathrm{a}}$ ed.). Madrid: McGraw-Hill.

Jackson, P. (1992). La vida en las aulas. Madrid: Morata.

Kuhn, T. (2004). La estructura de las revoluciones cientificas. México: Fondo de Cultura Económica.

Llopis, R. (2004). El grupo de discusión: Manual de aplicación a la investigación social, comercial y comunicativa. Madrid: ESIC. Recuperado de https://books.google.es/ books?id=ZuKMii2 TatcC\&printsec=frontcover\&hl=es\#v=onepage\&q\&f=false [Consultado el 5 de enero de 2017]. 
López, N. (2005). La Investigación Cualitativa: definición riesgos y posibilidades. En La investigación cualitativa: Nuevas formas de investigación en el ámbito universitario (pp. 15-27). Universidad Autónoma Latinoamericana.

Martínez, M. (2006). La investigación cualitativa (síntesis conceptual). Revista iipsi, 9(1), 126-146. Recuperado de https://es.scribd.com/ document/61302056/Martinez-Miguel-La-investigacion-cualitativa [Consultado el $1 .^{\circ}$ de enero de 2017].

Menda, A. M. y Méndez, J. M. (2009). La técnica de grupo de discusión en la investigación cualitativa. Aportaciones para el análisis de los procesos de interacción. Revista Iberoamericana de Educación, 49(3), 1-7. Recuperado de https://doi.org/https://doi.org/10.35362/rie4932094 [Consultado el 1. ${ }^{\circ}$ de enero de 2017].

Moral, C. (2006). Criterios de validez en la Investigación Cualitativa Actual. Revista de Investigación Educativa, 24(1), 147-164.

Murillo, G. (2014). La investigación biográfico-narrativa en educación. [Archivo de video]. Recuperado de https://www.youtube.com/watch?v=W7D1HS7ATkw [Consultado el 4 de febrero de 2017].

Organización de los Estados Americanos (OEA) y Agencia Interamericana para la Cooperación y el Desarrollo (AICD) (2005). La documentación narrativa de experiencias pedagógicas. Una estrategia para la formación de docentes. Ministerio de Educación Ciencia y Tecnología. Recuperado de https://www.unrc. edu.ar/unrc/academica/pdf/libro-narrac1.pdf [Consultado el 13 de enero de 2018]

Platón (2013). El Banquete. España: Editorial Alianza.

Pulido, R., Ballén, M. y Zúñiga, F. (2007). Abordaje hermenéutico de la investigación cualitativa: teorías, proceso, técnicas. Bogotá: Universidad Cooperativa de Colombia.

Roedig, A. (2012). El trabajo intelectual se degrada: aunque cada vez se produce más saber, son menos los que pueden vivir de su trabajo. Sinpermiso. Recuperado de http://www.sinpermiso.info/textos/la-educacin-es-perjudicial [Consultado el $1 .^{\circ}$ de enero de 2018].

Sandín, M. (2000). Criterios de validez en la investigación cualitativa: de la objetividad a la solidaridad. Revista de Investigación Educativa, 18(1), 223-242. Recuperado de http://revistas.um.es/rie/article/view/121561 [Consultado el 26 de diciembre de 2018].

Sandín, M. (2003). Tradiciones en la Investigación Cualitativa. En Investigación Cualitativa en Educación: fundamentos y tradiciones (pp. 141-184). Madrid: McGraw-Hill.

Schrodinger, E. (1998). Mi concepción del mundo. España: Tusquets Editores.

Schwab, J. (1996). The practical: A language of curriculum. The School Review, $78(1), 1-23$.

Stake, R. (2007). Investigación con Estudio de Casos. Madrid: Morata. 
Tadeu, T. (1999). Documentos de Identidad. España: Belo Horizonte.

Upegui, M. (2015). Demanda de profesionales en el mercado laboral como una aproximación a la pertinencia de la educación superior en Colombia. (Trabajo de grado). Universidad Eafit, Medellín. Recuperado de https://repository. eafit.edu.co/bitstream/handle/10784/8525/MariaVirginia_UpeguiMejia_2015. pdf? sequence $=2$ [Consultado el 12 de enero de 2018]. 


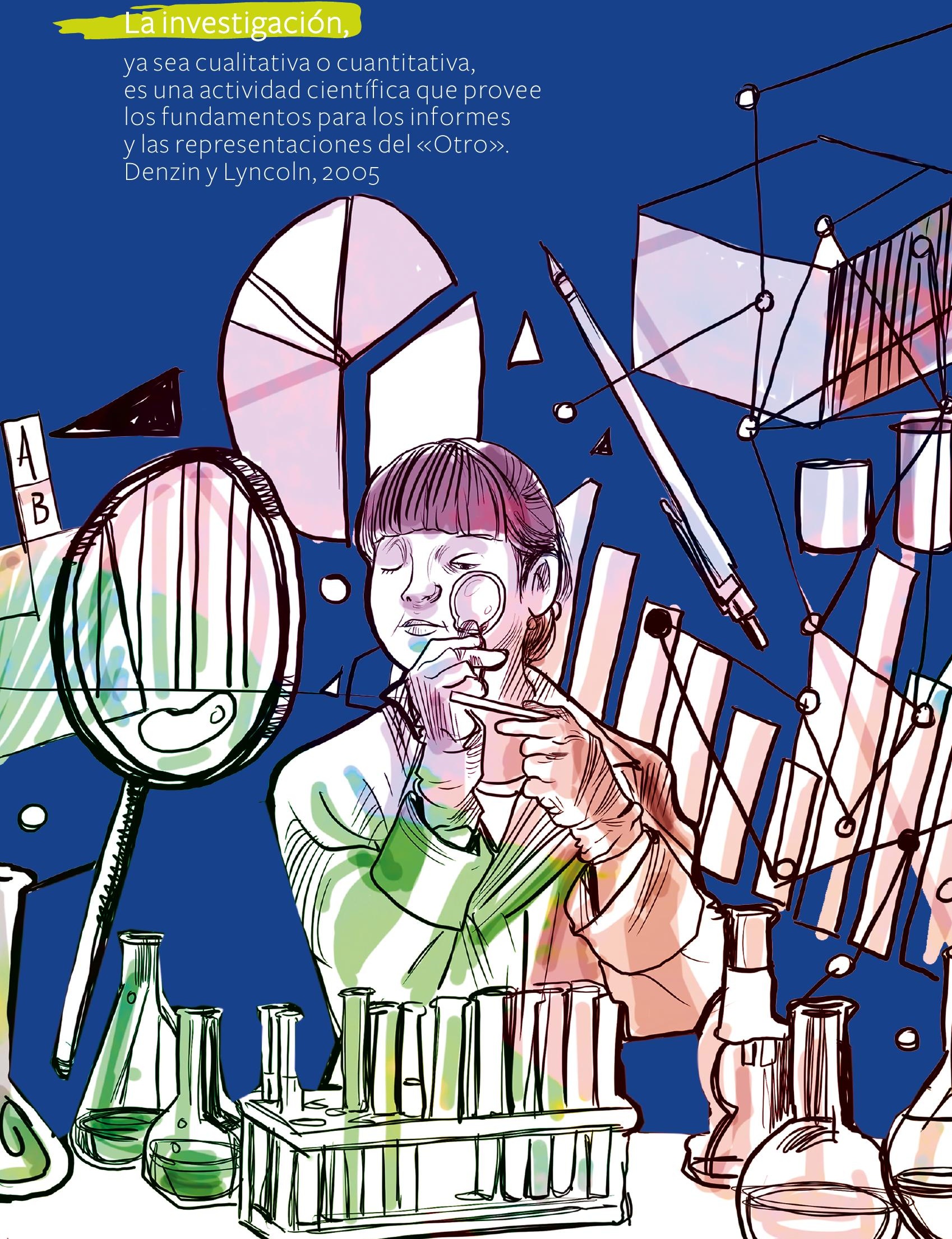

